\title{
Conflict of interest: forward not backward
}

\author{
Fiona Godlee editor in chief, The BMJ
}

The New England Journal of Medicine has refuelled the smouldering debate on conflicts of interest. In a surprising series of articles and an editorial by its editor in chief, Jeffrey Drazen, the journal seems to signal a retreat from current efforts to tackle financial conflicts of interests in medicine (doi:10.1056/ NEJMms1502493, doi:10.1056/NEJMms 1502497, doi:10.1056/ NEJMms1502498, doi:10.1056/NEJMe1503623).

There has been no shortage of critical response, including in The BMJ. In an editorial I and colleagues conclude that it's a mistake to suggest that rigorous standards should be revisited (doi:10.1136/bmj.h2957). And an accompanying article by three former editors of the New England Journal of Medicine, Robert Steinbrook, Jerome P Kassirer, and Marcia Angell, calls its series of articles "a seriously flawed and inflammatory attack" that tries to rationalise conflicts of interest in the medical profession (doi:10.1136/bmj.h2942). They fear a further weakening of conflict of interest policies at the New England Journal of Medicine and hope that its stance will serve as a wake-up call.

Other contributions to the debate come from bloggers surprised and concerned at the journal's new stance (http://1boringoldman. com/index.php/2015/05/24/a-narrative, http://hcrenewal. blogspot.co.uk/2015/05/say-it-aint-so-logical-fallacies-in.html, www.healthnewsreview.org/2015/06/nejm-reignites-conflictof-interest-debate-with-reader-poll). Indeed it's hard to find support for the New England Journal of Medicine's move. Richard Horton, editor of the Lancet, comes closest (doi:10. 1016/S0140-6736(15)61034-0). The truth, he says, lies somewhere between these extremes.

So where is this common ground? No one doubts the need for a vibrant drug and devices sector that serves patients and populations. Nor does anyone seriously question that, to deliver this, the industry must interact with researchers and understand the needs of patients and clinicians. And there is little dispute that non-financial conflicts of interest-such as academic passion and personal belief-are just as important, if harder to track.

Has the debate been useful? Horton thinks so, and I agree. Two clarifications in particular. Firstly, this is not a moral but a practical issue. As Steinbrook and colleagues say, it should not be insulting to suggest that a person's judgment can be affected by financial relationships. "The concern is not whether physicians and researchers who receive money have been bought by the drug companies ... The essential issue is that it is impossible for editors and readers to know one way or another."

Secondly, the same person or people shouldn't be asked to produce the evidence and appraise it. As our editorial says, "These are different professional responsibilities, and they clash." The BMJ's new policy on conflicts of interest among authors of educational articles seeks to make clear this distinction (doi:10.1136/bmj.g7197). Ironically, we took as our model the New England Journal of Medicine's former policy, which set similar rules. These are hard to implement, but we are determined to push on, evaluating as we go.

Our aim is not only to ensure that our educational content can be trusted but to encourage culture change in medicine in the interests of patients and the public. We seek experts in all fields of medicine who do not have relevant financial relations with the industry. If you are such a person and would like to contribute, please email us (editor@bmj.com) so we can add you to our growing database.

Cite this as: BMJ 2015;350:h3176

๑ BMJ Publishing Group Ltd 2015 